face voluntarily, without any difference between the two sides, on involuntary laughter he only smiles with the left side of his face, the opposite side being perfectly passive. He protrudes his tongue in the midline and can move it from side to side. There is no atrophy nor tremor. The jaw drops in the median line. The actions of the temporals and masseters are well preserved.

The arms are of good development. Power, by the grip test, is somewhat diminished in the left side. Resistance to passive movement is equally good on both sides. He can perform all the normal movements well. In the finger to nose test there is a marked ataxia on the left side, almost amounting to an intention tremor. In the right extremity no ataxia is apparent. When he extends his hands there is a coarse tremor of both hands, that in the left being much more marked than that the right. The oscillations are upwards and downwards. There is no tenderness over nerve trunks. Atrophy is absent. Biceps and triceps jerks are exaggerated, and equally on each side: The legs present a good musculature. All normal movements can be well performed. In the heel to knee test no ataxia is demonstrable in either extremity. No atrophy or tenderness is present over nerve trunks. Knee jerks and ankle jerks are exaggerated on both sides. Plantar stimulation causes plantar flexion of the toes on both sides. Ankle clonus is absent. The patient shows no alteration of station with eyes open or closed. He also exhibits no abnormality of gait with eyes open, but when closed incoördination is present to a slight extent.

In the right half of the body to the median line absolute loss of sensation to pain and temperature is present. In testing he calls the pin prick dull, except on the right side of the forehead, where he says he feels the sharp touch. Although he feels here, the sensation on the opposite side is much more acute. Touch and muscle sense seem to be well retained. There is no astereognosis. The sphincters are apparently normal. No defects of speech are noted. The mentality of the patient is good; no delusions nor hallucinations being present. The chest of the patient is well formed and he has good expansion on both sides. Pulmonary resonance is good throughout. On auscultation normal breath sounds are heard. No adventitious sounds present. Examination of the heart shows that the apex beat is not observed on inspection. On palpation it is found to be diffuse, although not forcible in the sixth interspace. Cardiac dulness is slightly enlarged. The first apex best is weak. No murmurs can be detected. The spleen and liver are not enlarged, and no tumors or ascites are apparent.

Dr. Spiller agreed with Dr. Mills in his diagnosis. He believed the lesion must be in or near the optic thalamus. Numerous cases are recorded in the literature of tmilateral ataxia on the side of a lesion in the brain stem, and tremor is believed to be a sign of a lesion in the cerebral peduncle, as in Benedikt's symptom-complex.

\title{
ASCENDING POSTEROLATERAL SCLEROSIS
}

\section{By T. H. Weisenburg, M.D.}

Some years ago Dr. Mills first described a new symptom complex which consisted in an ascending degeneration of the pyramidal tracts. Dr. Weisenburg now reports two cases in which there was an ascending 
degeneration not only of the lateral columns but also of the posterior. The first case was that of a man of 56 who seven years ago noticed a weakness and stiffness in the right lower limb which was followed in three years by a similar condition in the right upper limb. Two years ago, or five years after the beginning of his disease he developed a similar condition in the left lower limb. He now has a typical, spastic paretic gait with ataxia of both upper and lower limbs, more marked in the right side, increased reflexes and bilateral Babinski phenomenon. $\mathrm{He}$ has besides a disturbance of sensation for touch and pain in all the limbs, more marked in the lower. There is an uncertain history of syphilis with some pupillary inequalities.

The second case was a young man of 29 with no previous history who four years ago first noticed a stiffness in the left lower limb. This increased and two years afterward involved the left upper limb and for the past six months the right lower limb has become similarly involved. He complains of numbness in all of these parts and has disturbance of sensation for touch and pain in the same areas. The reflexes are everywhere exaggerated and the Babinski phenomenon is obtained on both sides. $\mathrm{He}$ is distinctly ataxic in all the limbs, more so in the lower and with the eyes closed will have a tendency to stagger.

In both of these cases there is a distinct history of a gradual involvement of the lateral and posterior columns, first in the lower part of the spinal cord or one side and then the upper and then on the other side of the cord. Evidently here we have an ascending posterolateral sclerosis. The etiology in these cases is rather difficult to determine. In the first case, as there was a history of syphilis, it might be the result of this, but it is curious that such systematic degenerations should result. In the second case there was no history of disease, but the fact that it occurred in a young man probably suggests a congenital weakness in these parts.

Dr. Mills said in regard to one of the cases of Dr. Weisenburg it would seem in some respects like a case of disseminated sclerosis with a tendency, as in some cases of this disease, ascending or descending, unilateral or bilateral, to some degree of systematization.

Dr. C. S. Potts said in reference to one of Dr. Weisenburg's cases, that it occurred to him that it resembled one of the atypical forms of multiple sclerosis. The fact that the patient had diplopia at one time would bear this out. Some years ago Dr. Potts reported a case which somewhat resembled this although the posterior columns were not affected. There was also some paralysis of the ocular muscles and a temporal atrophy of the discs. That case was regarded as one of probable multiple sclerosis, though it was never proven by autopsy.

Dr. Alfred Gordon said that in the last two weeks he had an opportunity to see a case of the type of ascending paralysis described by Dr. Mills. It was a child of eight, a boy. He had made an effort to bring him before the Society but failed, the mother living out of town and not caring to come in at night. The striking symptom is this: The child was taken a year and a half ago with sharp, severe pain in the right foot, the pain persisted for some time, then paralysis developed. The mother noticed that the child walked lame, then dragged the leg as in hemiplegia. Yet later, while paralysis persisted in the leg the child developed pain in the right arm, then shortly after that paralysis of the right arm set in. The mother also told Dr. Gordon that at the time the paralysis of 
the leg developed a nystagmus appeared. Recently Dr. Gordon examined the boy and found typical hemiplegic gait. He presented exaggerated knee jerk, Babinski's sign, the paradoxical sign, etc. When the patient is told to walk he presents the gait of. a hemiplegic individual. He also presents a very marked nystagmus for upward and downward movements and in a milder degree for lateral movements. The history of the case is very interesting from this standpoint, perphaps of pathogenesis 'of disease. The disease began with pain in the leg and then pain in the arm, when paralysis set in. Perhaps there is some connection between the two phenomena. In considering the pathogenesis of the disease a possible neuritis should also be thought of instead of referring the paralysis to motor tract involvement. The question of multiple sclerosis comes up. He did not present any other symptoms except the marked nystagmus which came on at the same time as the paralysis of the leg. The clinical picture of the case is that described by Dr. Mills originally-an ascending paralysis.

Dr. Weisenburg said that he had had multiple sclerosis in mind in both of his cases. The examinations, however, denoted no changes in the optic discs, no nystagmus, and most important of all the symptoms appeared in such regularity that it would be difficult to reconcile them with the diagnosis of multiple sclerosis.

\section{MENTALLY DEFECTIVE CHILDREN IN THE PUBLIC SCHOOLS}

\section{By Walter S. Cornell, M.D.}

The recognition of the presence of mentally defective children in the regular grade classes of the public schools is not of recent date, but official cognizance and effort to aid these children is comparatively so, and the work along these lines has been conducted in a scientific manner only in a few of our largest cities. The function of this paper is simply to record a number of cases which have recently come under notice without systematic effort to discover them, and all existing in four public schools. Dr. Cornell then described certain illustrative cases.

A consideration of this subject would not be complete without reference to the special classes for backward children in New York City. Here large numbers of such children have been gathered, those from the East Side displaying an especially low grade of mentality. Dr. Cornell recently visited one of these classes, organized in the public school at i6o Christy St. Of the eighteen children in the class, all had physical defects. Defective vision was possessed by every child, three were deaf and six had nose and throat defects. Malformed skulls were noticeable in the majority of cases, some showing hydrocephalus and still others asymmetry of the cranium. Defects in the motor apparatus were evident, many of the children showing not only weakness in gait but also a pronounced weakness in one limb, which unilateral weakness is rather common in actually mentally feeble children from cerebral paralysis or functional weakness.

-Dr. John K. Mitchell asked whether he were correct in understanding Dr. Cornell to state that chorea was commonly associated with mental defect. If so, whether other gentlemen present agreed with the statement. Dr. Mitchell's experience was directly opposed-that is that choreic children were rather unusually bright.

Dr. G. E. Price referring to the percentage of syphilis and tubercu- 